\title{
Acknowledgement to Reviewers of Veterinary Sciences in 2019
}

Veterinary Sciences Editorial Office

MDPI, St. Alban-Anlage 66, 4052 Basel, Switzerland

Published: 20 January 2020

The editorial team greatly appreciates the reviewers who have dedicated their considerable time and expertise to the journal's rigorous editorial process over the past 12 months, regardless of whether the papers are finally published or not. In 2019, a total of 106 papers were published in the journal, with a median time to first decision of 30 days and a median time from submission to publication of 51 days. The editors would like to express their sincere gratitude to the following reviewers for their generous contribution in 2019:

Abidu-Figueiredo, Marcelo

Achenbach, Jenna E.

Ahlstrom, Christina

Amos, William

Antipova, Veronica

Aparacio, Pedro Mayor

Aranaz, Alicia

Arechiga-Ceballos, Nidia

Awosile, Babafela

Azaizeh, Hassan

Azam, Aa Haeruman

Barlow, John

Bartlett, Paul

Bax, Carmen

Bazzano, Marilena

Bellini, Silvia

Bertazzolo, Walter

Bertelloni, Fabrizio

Beyhan, Sinem

Bezos Garrido, Javier

Bochniarz, Mariola

Bøgwald, Jarl

Bongiovanni, Laura

Borzacchiello, Giuseppe

Bovera, Fulvia

Bown, Kevin
Breeze, Beth

Britti, Domenico

Brown, David

Bueno-Cavanillas, Aurora

Bulatov, Emil

Bulysheva, Anna A.

Byrne, Andrew

Cai, Demin

Cambier, Carole

Cameron, Daniel

Cannon, Claire M.

Carrillo, Jeniffer

Cassidy, Joseph

Castiglioni, Bianca

Cerecetto, Hugo

Chen, Yi-Ning

Chenais, Erika

Cheng, Ke

Chochlakis, Dimosthenis

Clarke, Jonathan

Connor, Melanie

Cortese, Laura

Cortey, Marti

Crasta, Manuela

Cribb, Alastair E.

Cutrignelli, Monica Isabella 
Cwynar, Przemyslaw

Damiaans, Bert

Dance, David A. B.

Dandrieux, Julien R. S.

Dark, Michael

Datan, Emmanuel

Davail, Blandine

De Biase, Davide

De Briyne, Nancy

De Rooster, Hilde

Del Chicca, Francesca

Devriendt, Nausikaa

Dewell, Grant

Dias-Pereira, Patricia

Dkhar, Hedwin Kitdorlang

Domínguez Rebolledo, Álvaro Efrén

Dooley, Laura M.

Dorman, David C.

Douet, Jean-Yves

Drigo, Michele

Driver, Ashley

Dumetre, Aurélien

Duran, Sue Hudson

Duszka, Kalina

Dvorak, Cheryl

Eda, Shigetoshi

Eichwald, Catherine

Elmi, Alberto

El-Sheikh Ali, Hossam

Emam, Mahmoud Abdelghaffar

Fan, Jianglin

Fasina, Folorunso Oludayo

Fei, Andrew Chang-Young

Ferreira, Fernando

Fischer, Amy

Fonseca-Alves, Carlos Eduardo

Frossard, Jean-Pierre

Fthenakis, George

Gantenbein, Benjamin

Giacomo, Gnudi

Giger, Urs

Giovanardi, Davide

Gómez-Arrue, Javier
Goodla, Lavanya

Gordon, Jessica

Gordon, Stephen V.

Goyal, Rajni

Goy-Thollot, Isabelle

Goździewska-Harłajczuk, Karolina

Grant, Irene

Gunesekera, Amila

Hafez, Hafez Mohamed

Halos, Lenaig

Ham, Kathleen

Haney, Evan

Hariharan, Harry

Hasbun, Jaime

$\mathrm{He}$, Chend

He, Qigai

Hearn, Michael J.

Heller, Martin

Hersperger, Adam R.

Hertkorn, Kathleen P.

Heuer, Cord

Hildreth, Blake

Hildreth, Michael B.

Hine, Brad

Hofmeister, Erik

Hotea, Ionela

Hubková, Beáta

Huneau, Adeline

Ilyinskii, Petr O.

Inga, Alberto

Iorio, Ronald M.

Isoda, Norikazu

Jahns, Hanne

Jaja, Ishmael

Jansen, Christine A.

Jansson, Désirée

Jefferson, Wendy N.

Jensen, Kirsty

Jeyanathan, Jeyamalar

Jung, Dong-In

Kajamies, Anu

Kang, Seong

Kawaji, Satoko 
Kennedy, Richard B.

Keuling, Oliver

Kilcawley, Kieran

Kitajima, Shuji

Klotz, James

Knauer, Whitney

Koralur, Munegowda

Krockenberger, Mark

Kumar, Anand

Kupke, Alexandra

Laddomada, Alberto

Laham-Karam, Nihay

Lahbib-Mansais, Yvette

Lei, Zhihai

Leite, Fábio Pereira Leivas

Liao, Albert T.

Liegner, Kenneth

Linhares Martins Gabriel, Dra. Áurea

Lipschutz-Powell, Debby

Liu, Jingbo

Lo, Szecheng

Lopes, Ana Patrícia Antunes

López-Gatius, Fernando

Lujan, Lluis

Lunney, Joan K.

Lutta, Harrison Osundwa

Maglio, Melania

Mahony, Timothy John

Mantzoukas, Spiridon

Mao, Dagan

Maranesi, Margherita

Marc, Daniel

Marconato, Laura

Marletta, Donata

Martano, Marina

Martens, Ann

Martínez-Sánchez, Noelia

Martini, Filippo Maria

Massari, Federico

Materniak-Kornas, Magdalena

Mathews, Karol Ann

Matumba, Limbikani

Maurelli, Maria Paola
McCleary-Wheeler, Angela L.

Mcdonald, Jennider

McKinley, Trevelyan J.

McMurray, David N.

Mehlhorn, Heinz

Melendez, Pedro

Meling, Daryl D.

Merino, Eva Maria Perez

Meyer-Aurich, Andreas

Mila, Hanna

Mokhtaria, Kouidri

Moldal, Elena Regine

Moreau, Maxim

Moutelíková, Romana

Mullin, Christine

Neira, Victor M. N.

Nguyen, Frédérique

Nichols, Megin

Niedringhaus, Kevin D.

Nuttall, Patricia A.

Oblak, Michelle

Okumura, Hiroki

Ozawa, Makoto

Paes, Geert

Paixão, Tatiane Alves

Parambeth, Joseph Cyrus

Pavicich, Maria Agustina

Pelizzo, Gloria

Penrith, Mary-Louise

Pereira, Maria De Lourdes

Perrucci, Stefania

Perry, Michael J.

Peterson, Karianne Lievaart

Phalen, David

Plaza-Díaz, Julio

Pozzo, Luisa

Prosheva, Valentina

Provot, Thomas

Qiu, Hua-Ji

Raftery, Alexandra G.

Rampazzo, Antonella

Ranadheera, Seneka

Ravera, Ivan 
Reavill, Drury

Reed, Kevin F. M.

Reif, Kathryn E.

Reifinger, Martin

Ressel, Lorenzo

Riad, Bouzid

Riva, Federica

Robertson, Ian

Rönnberg, Henrik

Rose, Nicolas

Roura, Xavier

Rudd, Penny

Sacco, James

Sakkas, Hercules

Samy, Ahmed

Sanad, Yasser

Sandro, Rolesu

Sani, Marc-Antoine

Šarkan, Bojan

Saxena, Vikas

Schaefer, Michael

Schildgen, Olivier

Schmidt-Bleek, Katharina

Schoiswohl, Julia

Schotthoefer, Anna M.

Schwarz, Tobias

Scribante, Andrea

Secott, Tim

Seyfang, Jemma

Shil, Niraj

Shimada, Akinori

Shiratori, Hidetaka

Simulundu, Edgar

Singh, Pallavi

Sinha, Amit

Siniscalco, Dario

Slana, Iva

Smith, Dale

Smith, Ed

Snyder, Orman (Larry)

Sopena, Joaquín

Specht, Andrew

Stent, Andrew
Suárez, Cinta Prieto

Suarez-Vega, Aroa

Sultan, Khaled

Sun, Kunfeng

Swelum, Ayman

Szóstek-Mioduchowska, Anna

Szulczyński, Bartosz

Tajima, Atsushi

Takeshima, Shinnosuke

Takizawa, Naoki

Tashima, Toshihiko

Tedesco, Idolo

Thaler, Robert

Thapa, Santosh

Thompson, Nathanael

Tibor, Magyar

Toledo, Alvaro

Tomaszewska, Ewa

Toomer, Gabriela Chavez-Calvillo

Tothova, Csilla

Tsairidou, Smaragda

Tufarelli, Vincenzo

Ventrella, Domenico

Verguts, Jasper

Vezzosi, Tommaso

Villari, Sara

Vrentas, Catherine E.

Wales, Andrew

Watkins, Craig

Weiss, Mark L.

Wendelburg, Kristin

Whelan, Stephen

White, Nathaniel A.

Whittington, Richard J.

Wojtyczka, Robert

Wolf, Peter

Wooton-Kee, Clavia Ruth

Wu, Jack H. Z.

$\mathrm{Wu}$, Meng-Yu

Wycislo, Kathryn

Xie, Qingmei

Yang, Run-jun Yang

Zaleski, Halina 
Zani, Laura

Zappulli, Valentina

Zarogoulidis, Paul

Zhalniarovich, Yauheni
Zhang, Guanshi

Zhao, Shaying

Zmora, Pawel

Zou, Wei

() 2020 by the author. Licensee MDPI, Basel, Switzerland. This article is an open access article distributed under the terms and conditions of the Creative Commons Attribution (CC BY) license (http://creativecommons.org/licenses/by/4.0/). 